\title{
Role of Artificial Intelligence in Medical Imaging for Accelerated Response in Dual Pandemics of TB and Covid-19
}

\section{Amit K* \\ Professor of Radiology, Dr DY Patil University, India}

*Corresponding author: Amit kharat, MBBS DMRD DNB PhD FICR, Professor of Radiology, DPU, Co-Founder, Dr DY Patil University, India, Tel: 9890046766; Email: amit.kharat@dpu. edu.in

\section{Mini Review}

Volume 5 Issue 1

Received Date: April 27, 2021

Published Date: May 12, 2021

DOI: $10.23880 /$ phoa-16000184

\section{Abstract}

The world is facing dual pandemics of TB and Covid-19 simultaneously. Digital Chest X-ray is the mainstay for screening chest TB. In community setting for TB screening, its likely that Covid-19 and similar community acquired pneumonia are likely to be detected. The key milestones of TB elimination need recalibration under the shadow of Covid-19 pandemic. Technological innovations like deep learning or computer assisted detection (CAD) can assist in instant screening and triage for TB in such situations. Innovative solutions like these have the potential to reduce the burden on the healthcare ecosystem and ensure access to healthcare imaging to the global population. Organizations will need to think through well while deploying and integrating these solutions seamlessly in the existing workflows. Organizations should understand the challenges AI solutions face and have safeguards to mitigate these risks. Reliable technological solutions are the best hope mankind has to empower experts and ensure imaging is accessible, affordable and available to all.

\section{Mini Review}

In population health management, TB figures as a critical disease condition where digital chest X-rays play a pivotal role in disease assessment and management. A key milestone for End TB Strategy is achieving around 95\% decline in deaths due to tuberculosis from a projected 110 cases/100 000 in 2015 to 10 cases/100 000 or less by 2035 [1]. At the other end of the spectrum is Covid-19, a corona virus infection. The covid pandemic has brought the world to a standstill as most of the countries have been following a complete lockdown to curb the infection, where people isolate themselves at home and socialization of any type is discouraged.

Digital chest X-ray of has been illustrated to be crucial in diagnosis and management of TB. Its role in TB screening program is well established. The typical appearance of TB on chest $\mathrm{x}$-ray is characteristic with its various patterns. It may manifest as pneumonia like areas of consolidation typically involving upper and middle lobes with or without hilar lymphadenopathy. Other radiological features of active primary TB are miliary opacities, pleural effusion or bronchoalveolar pattern [2]. Digital chest x-rays have been performed for TB screening in hospital-based setting or even remotely in periphery through digital mobile vans equipped with portable digital $\mathrm{x}$-rays machines. TB screening from digital chest $\mathrm{x}$-ray can therefore happen in hospitals, clinics or even mobile vans.

For Covid-19 pneumonia, hospital-based screening and follow up assessment especially in the ICU there is role for bedside portable examination like Xray of the chest for 
understanding lung health and response to treatment. For Covid-19 it may not just be a potential diagnostic tool, but also can support in evaluating the trajectory of disease progression as well as in predicting outcomes when correlated with other parameters. On digital chest x-ray the covid-19 pneumonia is typically seen as airspace opacities either consolidations or ground glass opacities (GGO) even at an early stage [3]. The abnormalities are often bilateral, peripheral and prominent in the lower zone [4]. Moreover, the radiographic abnormalities may not necessarily correlate with the clinical symptoms. Covid screening therefore is likely to happen in hospital settings or may be incidentally encountered in community mobile van-based setting for TB screening.

Since the covid-19 surge, medical imaging has been focused around Covid-19 to ensure speedy delivery of care. It has resulted in serious setback to targets of almost all nations for TB screening and it's likely that governments may have to recalibrate goals on TB elimination. Also, the multiple waves of Covid-19 infections due to its various mutations, makes it difficult to estimate how long it will take to find a way to eliminate Covid-19.

Imaging experts are significantly burdened globally due to high numbers of scans happening daily and it has been over whelming. How can technology are used in management of two pandemics to improve efficiency. The new age technology of Artificial Intelligence (AI) based prescreening and triage using point deep learning solutions looks promising. Using an AI embedded end to end imaging platform, which assists imaging experts from reaping the benefits of prescreening, provide structured radiology report and also use the powerful analytic tool is a potential solution.

Various clinical research and audits done by WHO and various clinically validated research papers establish key role of point solutions for TB screening and covid triage respectively. Through a recent communication by WHO with respect to TB, it states "Computer Aided Detection (CAD) can be may be used as an alternative to human reader interpretation of plain digital CXR for screening and triage for TB. Its use should be limited to the interpretation of plain CXR for pulmonary TB in individuals aged 15 years or more" [5]. The problems caused by COVID-19 infection in a population already burdened with TB are multi-faceted. TB programs globally have hit a road block due to the emergence of COVID-19.
AI based instant triage and prescreening offers hope to optimize workflows in radiology department in government hospitals, private care centers and portable mobile digital $\mathrm{x}$-ray screening centers, by allowing appropriate rationale utilization of scare and costly resources and use the powerful dashboard for key disease insights. Such platforms can serve dual purpose like prescreening and provides instant triage especially in situation of low resource setting. Multiple AI algorithms can be combined which can allow for screening of community acquired pneumonias and TB and various other conditions in the same setting. The output can also be quicky audited by an expert if needed.

Many nations have shown initial proof of concept through such unique deployments. STOP TB through its role in auditing these solutions has presented its research, new guidelines and framework to various nations on its usage in existing workflows [6]. These are likely to benefit TB screening programs under the shadow of the Covid pandemic.

Though AI has its merits we cannot ignore challenges during implementation such as data drift and model decay. With adequate safeguards with an expert in the loop and regular audits this can be certainly tackled. Finding outlier studies is also a challenge that needs to be carefully understood and taken care off.

It seems that AI will play a significant role in all aspects of medical imaging for population screening and it will have far reaching impacts. The x-ray of chest in its digital avatar continues to bring great insights and significantly impacts the screening, diagnosis and management for TB and to some extent COVID. With AI solutions applied on top of it either as edge solution or cloud offering makes the impact quite strategic allowing nations to use new age technology to empower experts to efficiently respond to pandemics. As new insights are gathered, their role and workflow will keep getting redefined based on the location such solutions will be deployed, such as within the modality itself, such as portal X-ray machines (edge) or on PACS or cloud setting. It will also impact the process of image generation, pre and post processing. AI will potentially enhance the experts, decongest their radiology work flow, minimize the stress, and eliminate repetitive tasks and thereby allowing experts to focus their attention on critical issues at hand. Reliable technological solutions are the best hope we have to empower experts and ensure imaging is accessible, affordable and available to all (Figure 1). 


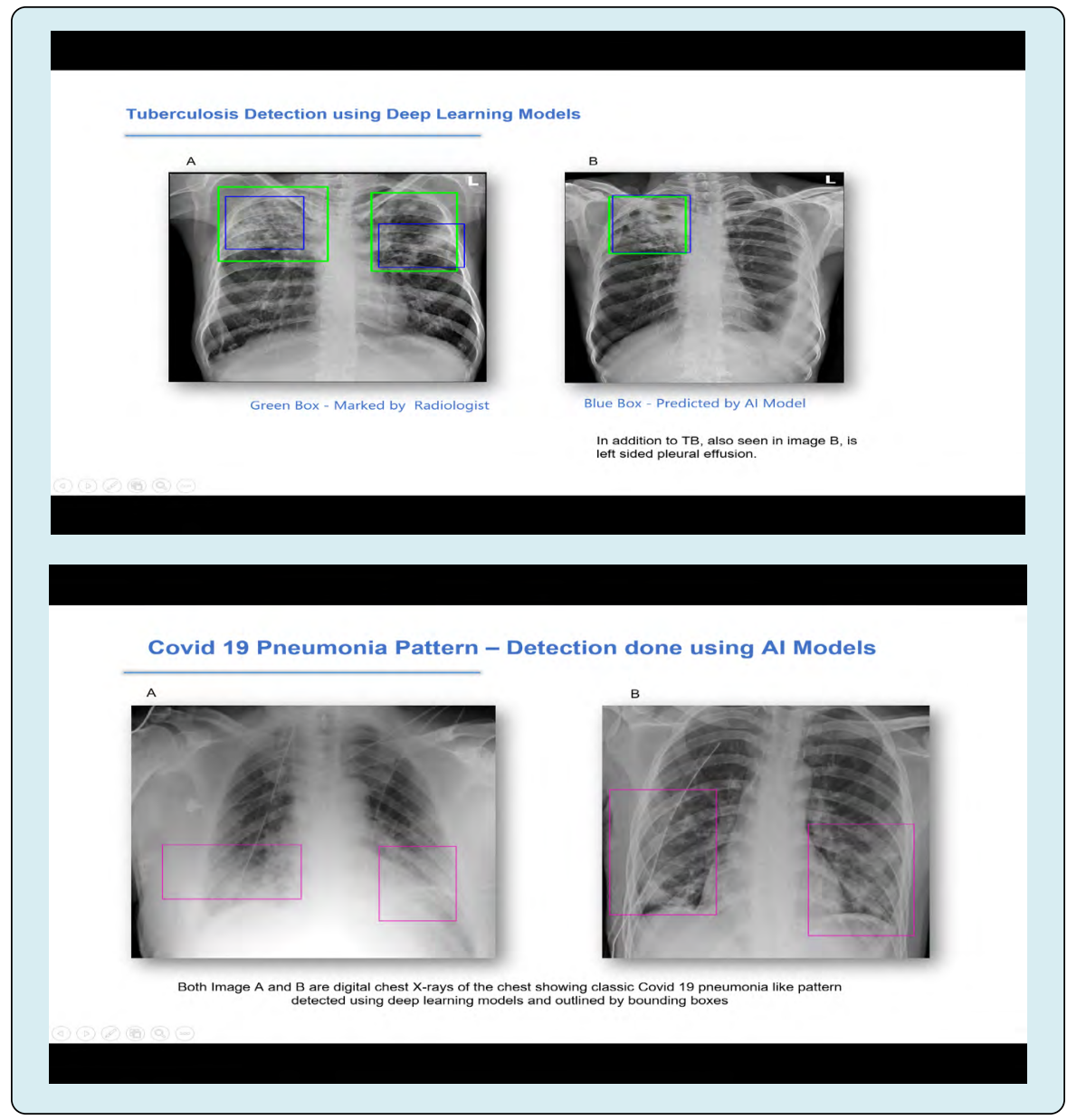

\section{References}

1. WHO (2021) Rapid communication on the systematic screening for tuberculosis. World Health Organization.

2. Al Ubaidi BA (2018) The Radiological Diagnosis of Pulmonary Tuberculosis (TB) in Primary Care. J Fam Med Dis Prev 4(1): 7.

3. Aguiar ERGR, Navas J, Pacheco LGC (2020) The COVID-19 Diagnostic Technology Landscape: Efficient Data Sharing Drives Diagnostic Development. Front Public Health 8:
309.

4. Durrani M, Haq IU, Kalsoom U, Yousaf A (2020) Chest X-rays findings in COVID 19 patients at a University Teaching Hospital - A descriptive study. Pak J Med Sci 36(COVID19- S4): S22-S26.

5. WHO (2020) Rapid communication on the systematic screening for tuberculosis. World Health Organization.

6. (2021) AI-Powered Computer-Aided Detection (CAD) Software. 